\title{
The Assessment Screening of Trauma Reactions, Psychopathology and Psychosocial Function in a High-Risk Sample of 4 - 8-Year-Old Children-A Pilot Study
}

\author{
Irene Marcela Jørgensen ${ }^{1 *}$, Cathriona Cantio ${ }^{2}$, Ask Elklit ${ }^{1}$ (]) \\ ${ }^{1}$ Danish National Centre of Psychotraumatology, Department of Psychology, University of Southern \\ Denmark in Odense, Odense, Denmark \\ ${ }^{2}$ Department of Psychology, University of Southern Denmark in Odense, Odense, Denmark \\ Email: *marcelairene2@gmail.com
}

How to cite this paper: Jørgensen, I.M., Cantio, C. and Elklit, A. (2019) The Assessment Screening of Trauma Reactions, Psychopathology and Psychosocial Function in a High-Risk Sample of 4 - 8-Year-Old Children-A Pilot Study. Open Journal of Epidemiology, 9, 15-31.

https://doi.org/10.4236/ojepi.2020.101002

Received: October 16, 2019

Accepted: December 10, 2019

Published: December 13, 2019

Copyright $\odot 2019$ by author(s) and Scientific Research Publishing Inc. This work is licensed under the Creative Commons Attribution International License (CC BY 4.0).

http://creativecommons.org/licenses/by/4.0/

(c) (i) Open Access

\begin{abstract}
Background: Multiple traumatization in childhood is a broad term that includes interpersonal trauma experiences such as physical, sexual, and emotional abuse and neglect. Children with a child protection case referred to counseling are likely to be in high risk of multiple exposure to interpersonal trauma. We aim to demonstrate that systematic assessment with validated measures is feasible in this age group. Method: The Center for Interventions of Children and Adolescents (CIBU), Denmark, provided a new assessment screening procedure where 16 caregivers with children in the age of 4 - 8-years-old participated prior to initiating counseling in a public family treatment facility. We utilized the Diagnostic Infant Preschool Assessment (DIPA) and the Strengths and Difficulties Questionnaire (SDQ). The SDQ was compared with Danish norms. Results: The data suggest that psychopathology was present among all 16 children in this high-risk sample. Distribution of disorders showed $93.8 \%$ prevalence of comorbidity. All 16 children had difficulties regarding psychosocial functioning when compared to a $\mathrm{Da}$ nish norm population. Most profound was emotional symptoms and symptoms of hyperactivity and inattention. Conclusion: This study revealed a higher prevalence of psychiatric disorders and higher rates of psychosocial difficulties in referred children than in children from the general population. Overall, children in this study had complex symptom profiles. Thus, a systematic approach may be helpful in public treatment facilities, and we suggest the implementation of valid evidence-based instruments.
\end{abstract}




\section{Keywords}

Multiple Traumatization, Young Children, Child Protection Case, Assessment, DIPA, SDQ

\section{Introduction}

It is estimated that two out of three children will experience at least one potential traumatic event before the age of 16 [1]. Severe trauma and child adversity seem to shape the individual vulnerability and often precede psychopathology within and across diagnostic boundaries [2] [3]. However, even after experiencing traumatic events, many children display resilience and do not develop enduring trauma symptoms, while others seem to be particularly vulnerable and develop psychopathology after trauma and childhood adversity. Several factors, including developmental stage, amount of social support, attachment style and intelligence level may influence which children develop psychopathology after exposure to traumatic events [4].

Various studies have shown that children exposed to child adversity present a wide range of internalizing and externalizing problems. Adverse childhood experiences can shape the individual vulnerability and often precede psychopathology within and across diagnostic boundaries [3]. Early adversity and traumatic experiences exert powerful effects on the brain, body and psychological development lasting throughout the entire life span, influencing brain function and increasing vulnerability to stress, depression, and anxiety disorders [2] [5] [6].

There is an ongoing debate whether the current diagnostic criteria for Post Traumatic Stress Disorder (PTSD) are appropriate for all children because it does not distinguish between acute trauma (exposure to a single overwhelming event) and chronic or multiple traumas such as neglect of care and maltreatment over years [7]. Additionally, traditional diagnostic categories such as PTSD are argued to be limited since exposure to traumatic stress in early life is associated with enduring symptoms that not only are incorporate, but also extend beyond PTSD. Further, the assessment of children with a history of complex developmental trauma presents a significant challenge to services [8].

In 2008-2009, a Danish random probability survey sample study was conducted. The data suggested that severe traumatic events rarely happen in isolation and that many of the particularly stressful incidents and interpersonal traumas often occur inter-related in a small, vulnerable group of children [9]. The study found that child protection status significantly increased an individual's probability of being exposed to emotional abuse, sexual abuse, and multiple abuse when compared to those not defined as child protection cases. A child protection is defined as an intervention the municipality does due to concern for the welfare of a child. It can range from minor supportive initiatives to removal 
of the child from the home. This poses tremendous demands on the public treatment facilities in how to asses and treats the psychopathology of these children.

Recently, a Danish study examined the onset of seven psychiatric disorders and the severity of functional impairment in preschool children following different kinds of traumatic events. The results suggested that about half of the children suffered from PTSD and comorbid disorders with oppositional defiant disorder being the most common [2]. The authors concluded that psychiatric assessments across numerous domains are essential before treatment planning. However there is still a need for additional research since the understanding of comorbidity among preschool traumatized children is limited and fragmented [2] [10]. Furthermore, many of the known studies in the field have only examined psychopathology in young children exposed to single trauma events. The studies are therefore not representative of the many types of repeated trauma a young child can experience, such as neglect, sexual, emotional or physical abuse, despite research indicating that repeated exposure to interpersonal trauma is associated with more profound and more complex trauma reactions [11] [12].

The ages between 4 and 8 years are of particular importance for identifying patterns and predictors of psychopathology and malfunction, as children navigate critical developmental tasks around the transmission and adaption to school [13]. In Denmark, most children begin in school at the age of six. Starting school is characterized by increased demands on the child regarding cognitive and academic, as well as social and practical development. From a neurocognitive perspective this phase of a child's life constitutes a very rapid cognitive development [14] [15]. Therefore, there is a great need for careful assessment and intervention for this age group of children.

Assessment and treatment of trauma reactions and psychopathology in young children require special considerations due to developmental differences in cognitive, language, emotional, and behavioral capacities [16]. Structured diagnostic interviews, which have evolved along the development of classification systems, are now widely used in child and adolescent mental health services as well as in the fields of clinical trials, epidemiological studies and academic research. However, despite the need of thorough assessment there is no golden standard in terms of utilization of structured diagnostic interviews and standardized measures in clinical practices such as public family treatment facilities. In a study from Norway [17], the authors investigated how management of child abuse could be further improved by mental health services. The study revealed that psychosocial functioning was seldom documented and that psychological symptoms should be described more detailed and reliably. Thus, the authors concluded a need for a systematic approach and implementation of valid evidence-based instruments in the field. However, the symptomatology, traumatizing and comorbidity appears not to have been explored systematically in a sample of 4 - 8 aged children referred to counseling by child protection. 
Considering these limitations, the present study aims to build on and extend the extant research by utilizing a clinical sample of Danish 4 - 8 aged children with a child protection case who initiated counseling. Until recently, diagnostic instruments did not exist for children under the age of 7. However, the Diagnostic Infant and Preschool Assessment (DIPA) is a well-validated assessment tool which can be used for this purpose [16]. DIPA is a semi-structured diagnostic interview with the caregiver about the child. We interviewed the caregiver to ascertain the amount of symptomology and functionality for the child in clinical domains. We also used the Strength and Difficulties Questionnaire (SDQ) [18] to assess the children's adjustment levels and level of function. The overall objective was to replicate the findings of [2] in a clinical sample of children who had an extended age range from $4-8$ years old.

\section{Methods}

\subsection{Participants}

The study included 16 children between the ages of 4 and 8 years old who initiated counseling in two community treatment facilities from the period of April 2017 to October 2017. Two treatment facilities served as an outpatient offer as a part of the Center for Interventions of Children and Adolescents (CIBU) in Odense, Denmark. The children and their caregivers were referred to counseling as a part of an ongoing child protection case. In this study, the children themselves did not participate directly. Participants consisted of caregivers who were willing to complete an interview and a questionnaire about their child as a part of a new assessment procedure in the treatment facilities. The caregiver could either be a parent or a foster parent.

\subsection{Measures}

\subsubsection{The Diagnostic Infant Preschool Assessment}

DIPA is a semi-structured interview with caregivers about their child. It includes all symptoms of the following 13 disorders according to the Diagnostic and Statistical Manual of Mental Disorders, fourth edition (DSM-IV): Attention Deficit Hyperactivity Disorder (ADHD), Oppositional Defiant Disorder (ODD), Conduct Disorder (CD), Major Depressive Disorder (MDD), PTSD, Separation Anxiety Disorder (SAD), Generalized Anxiety Disorder (GAD), Obsessive-Compulsive Disorder (OCD), social phobia, specific phobia, reactive attachment disorder (RAD), and sleep disorder. All diagnostic modules consist of several categorical questions concerning different kinds of psychiatric symptoms based on observable behavior. Each question begins with a stem question, which the interviewer reads verbatim. After a stem question, the interviewer uses his/her judgment on whether follow-up probes are needed. Interviewers must be a professional psychologist or otherwise trained employee in order to judge and enhance diagnostic accuracy. Interviewers can probe additionally until they feel satisfied that a symptom is present or not. Most importantly, interviewers are instructed to get 
an example of every symptom to verify respondents' answers with real examples. The DIPA assesses functional impairment in a disorder-specific fashion by asking about impairment at the end of each disorder. Five areas of role functioning (with parents, with siblings, with peers, at school/day care, and in public) plus a sixth item of child distress (except for ADHD and ODD) are assessed. The DIPA is a well-validated assessment tool and it is one of the few existing developmentally sensitive and time efficient methods to measure psychiatric disorders in preschool children [16] [19].

If the child being assessed was under 7 years old, PTSD was diagnosed by the alternative algorithm for young children (PTSD-AA; Scheeringa, Zeanah, Myers, $\&$ Putnam, 2003), because studies suggest that existing diagnostic criteria need substantial modifications to be valid for young children [10] [11] [20] [21] [22]. The main difference in the alternative algorithm compared to the DSM-IV-TR is that a verifiable reaction immediately after the trauma is not required. Additionally, only one of the seven symptoms in criterion $\mathrm{C}$ (avoiding and numbing symptoms) is required. Most of the main differences are comparable to the criteria for preschool PTSD presented in the newest edition of the DSM (DSM-5) [23] and have been empirically validated [2] [16] [19]. Furthermore, the diagnostic algorithm for MDD included the empirically validated developmental modification that sad mood and diminished interest in significant activities is considered symptomatic if the symptoms were present at least eight days out of two contiguous weeks [2] [24]. This is opposed to the requirement of nearly every day presented in the DSM-IV-TR [2].

\subsubsection{The Strength and Difficulties Questionnaire}

SDQ is a brief, behavioral, five factor instrument developed to assess emotional and behavioral problems in children and adolescents [18]. The SDQ contains 25 questions about different positive and negative aspects of the child's behavior and it includes impact supplement. Responses are made on a three-point Likert scale; "not true $=0$," "somewhat true $=1$ " and "certainly true $=2$ ". The items are divided into five subscales (hyperactivity scale, emotional symptoms scale, conduct problem scale, peer problem scale and prosocial scale) each comprising five items. The first four scales are summed up into the Total Difficulties Scale with a score that ranges from $0-40$. The prosocial scale is included to enhance acceptability on part of the rater. The impact supplement provides an important estimate of the burden of the problems which is an essential part of the diagnostic criteria in the current diagnostic classification systems, ICD-10 and DSM-IV. The Danish SDQ norms of children aged 5 - 7 that included data from three general population-based, large-scale birth cohorts were used for comparisons [25] [26]. In line with previous research the Danish norm data and cut-off-scores used as comparison in this study were provided by the SDQ website (http://www.sdqinfo.com/norms/DanishNorms.html; [25] [26]). The SDQ has good reliability and validity [27]. It has been translated into more than 60 languages. It is frequently used worldwide as a child mental health screening 
measure, for both children at large and for vulnerable populations [28] [29].

\subsection{Procedure}

Interviews were conducted in two community treatment facilities in the CIBU from April 2017 to October 2017. All caregivers with children between the ages of 4 and 8 years who initiated counseling during this period were invited to participate in the study. At the introduction meeting before the beginning of counseling, all caregivers received information about the research project and were invited to participate. Participation was voluntary and their decision did not influence on their course of treatment and the child protection case. Terms of confidentiality were presented verbally, however, it was also clarified that in case of findings of unreported abuse, information about the child would be handed to the authorities. Verbal and informed consent was obtained from the participants and dates for the participation were scheduled.

The caregiver could either be a parent or a foster parent that knew the child well. Prior to the interview, sociodemographic information about the child was collected including age, gender, known diagnoses, as well as whether the child was currently placed in foster care. The interviewers comprised of two graduate psychology students who were former trainee students at the treatment facility and had completed a course regarding the administration of DIPA and general interviewing techniques. Furthermore, in order to ensure the validity of the data the interviewers received clinical supervision in the process of collecting and administer the DIPA-interviews. All participants completed the SDQ questionnaire on the same day that they were interviewed with the DIPA.

After the completion of DIPA and SDQ relevant case files in every child protection case were screened for potential unreported traumas or suspicion of such incidents which the caregiver had not mentioned in the interview.

The preliminary data processing involved anonymizing the data. Afterward, all the children's psychological profiles were analyzed individually to clarify the categorical incidents of the different disorders according to the previously described algorithms. The project was carried out in accordance with the common ethical rules for Nordic psychologists and approved by the IRB. The data were stored securely at the Danish National Center for Psychotraumatology and we followed the GDPR data surveillance regulations for research projects.

\subsection{Data Analysis}

Analyses were carried out using the Statistical Package for Social Sciences SPSS 22 and were conducted on unweighted data. Descriptive analyses were performed to show the distribution of age, gender, type of trauma, the total number of symptoms, and the prevalence of the disorders across the sample. The percentage of parents reporting emotional and behavioral difficulties in their children was calculated according to the four subscales and the total difficulties score was obtained [25] [27]. 


\section{Results}

\subsection{Demographic Data}

The total study sample included $N=22$ families who initiated counseling during the period of data collecting. Three caregivers declined to participate in the study and furthermore three families were excluded from the study since the caregiver was unable to speak Danish at a functional level. The final sample was composed of 16 children $(N=16$, response rate $=76.2 \%)$. This sample had a mean age 6.3; SD $1.4 \pm$ years; range 4.1 to 8.3 years. There were 9 girls (56.3\%) and 7 boys (43.9\%). Further, all children meet the criteria for one or more mental disorder with a mean of 3.8 diagnoses per child, (SD 1.7, range $1-7$ ). The mean of the SDQ total difficulties score for this sample was 19.06 (SD 4.94, range 10 - 27).

\subsection{Characterization of the Type of Trauma}

All the children had been exposed to several traumas with a mean of 5.6 trauma experiences per child (SD 1.9, range from 3 to 9). The mean age for experiencing the first trauma was 1.8 years of age (SD 1.9). The different types of traumas are listed in Table 1. The most common traumatic experience categorized in the DSM-IV list was witnessing interpersonal violence and the least common experienced trauma was severe burn injury. Importantly, results indicated that all 16 children had been exposed to other forms of trauma not described by the categories. The types of trauma were either noted by the caregiver or it was revealed by the screening procedure of the child protection case files. The two most common traumas not specified were neglect of care and separation trauma.

\subsection{DIPA: Distribution of Disorders}

The rates and frequencies of diagnoses for the 11 diagnosed disorders are listed in Table 2. First, psychopathology was very common among the children in the study. All 16 children displayed enough symptoms to meet the criteria for at least one psychiatric diagnosis. The mean of diagnoses per child was 3.8; (SD $1.7 \pm$ and range $1-7)$. Nearly, $93.8 \%(N=15 / 16)$ of the children in the study met the criteria for three or more comorbid disorders. 5 (31.3\%) caregivers reported that their child met the criteria for four or more disorders. Only one caregiver reported that their child only displayed symptoms enough to meet the criteria for sleep disorder as the only psychiatric disorder.

The most common disorders were PTSD and ADHD inattentive subtype with a prevalence of $68.8 \%(N=11)$ followed by ODD $(50.0 \%, N=8)$ and RAD $(43.8 \%, N=7)$. No children met the criteria for bipolar affective disorder, GAD and OCD.

\subsection{SDQ Scores in Comparison to Norm Data}

The SDQ scores reported by each caregiver were analyzed for the severity of problems according to the four subscales the hyperactivity/inattention, emotional 
Table 1. Frequencies and counts of trauma types.

\begin{tabular}{|c|c|c|c|c|c|}
\hline $\begin{array}{l}\text { Type of DSM-IV } \\
\text { specified trauma }\end{array}$ & $\mathrm{N}$ & $\%$ & Other types of trauma & $\mathrm{N}$ & $\%$ \\
\hline Other & 16 & 100.0 & Neglect of care & 14 & 87.50 \\
\hline Witnessing violence & 11 & 68.8 & Separation trauma & 8 & 50.0 \\
\hline Medical trauma & 7 & 43.8 & Psychological abuse & 7 & 43.8 \\
\hline Physical abuse & 6 & 37.5 & $\begin{array}{l}\text { Child lives with a parent who } \\
\text { suffers from mental disorder }\end{array}$ & 5 & 31.3 \\
\hline Sexual abuse & 4 & 25.0 & $\begin{array}{l}\text { Severely high conflict level } \\
\text { between parents }\end{array}$ & 4 & 25.0 \\
\hline Traffic accident & 2 & 12.5 & Living in foster care & 4 & 25.0 \\
\hline Hurricane & 2 & 12.5 & Physical neglect & 3 & 18.8 \\
\hline $\begin{array}{c}\text { Close to drowning } \\
\text { accident }\end{array}$ & 2 & 12.5 & Severe bullying & 3 & 18.8 \\
\hline Abduction & 2 & 12.5 & $\begin{array}{l}\text { Child lives with a drug or } \\
\text { alcohol abusive parent }\end{array}$ & 3 & 18.8 \\
\hline Severe burn injury & 1 & 6.3 & $\begin{array}{l}\text { Sudden death of a close } \\
\text { family member }\end{array}$ & 2 & 12.5 \\
\hline- & & & Parent in prison & 2 & 12.5 \\
\hline- & & & Adoption & 1 & 6.3 \\
\hline- & & & Stalking of a caregiver & 1 & 6.3 \\
\hline
\end{tabular}

symptoms, conduct problem, peer problem and then summed to obtain a total difficulties score yielding a possible score ranging from 0 to 40 . The mean of the SDQ total difficulties score for the children in this study was 19.06 (SD 4.94, range 10 - 27) placing the mean within the clinical range. The SDQ scores in this sample were compared with normative SDQ frequency distribution scores for Danish 5 - 7-year-old girls and boys. Cutoff scores are listed in Table 2 and were based on [25]. To compare the item score characteristics of our Danish high-risk sample, we also present the item score characteristics of the Danish norm data sample by [25]. The Danish norm data sample followed Goodman's recommendations with approximately $80 \%$ of children defined as being within a "normal" range, followed by $10 \%$ in a "borderline" range and the highest $10 \%$ grouped in an abnormal or clinical range [18].

All 16 caregivers in this high-risk study sample rated their child's total difficulties scores above normal cut-off range when compared to the Danish norm population. 15 caregivers rated their child in the clinical range and one caregiver rated the child within the borderline range. The total difficulties score was 21.43 (SD 3.21) for boys and 17.22 (SD 5.43) for girls. Most children displayed emotional symptoms with a mean of 5.89 (SD 1.35) for boys and 6.22 (SD 2.39) for girls that means $75.0 \%(N=12)$ of the children had a clinical emotional problem score and further two scored within the borderline range. Problems of hyperactivity/inattention were also evident with a mean of 8.43 (SD 1.27) for boys and 4.88 (SD 3.22) for girls corresponding to $13(81.2 \%)$ caregivers rating their child within clinical or borderline range in the hyperactivity/inattention domain. 
Table 2. Distribution of mental disorders according to Diagnostic and Statistical Manual of Mental Disorders, Fourth Edition (DSM-IV) and PTSD-AA [16] [20].

\begin{tabular}{|c|c|c|c|}
\hline Disorder & $\begin{array}{l}\text { Total number of children } \\
\text { meeting the criteria }\end{array}$ & DSM-IV & PTSD-AA \\
\hline \multirow{2}{*}{ Sleep disorder } & $N=14$ & $N=1$ & \multirow[b]{2}{*}{ - } \\
\hline & $87.5 \%$ & $6.3 \%$ & \\
\hline \multirow{2}{*}{ PTSD } & $N=11$ & $N=7$ & $N=10$ \\
\hline & $68.8 \%$ & $43.8 \%$ & $62.5 \%$ \\
\hline \multirow{2}{*}{ ADHD } & $N=11$ & - & \multirow{2}{*}{ - } \\
\hline & $68.8 \%$ & - & \\
\hline \multirow{2}{*}{ ADHD (inattentive subtype) } & $N=10$ & $N=10$ & \multirow{2}{*}{ - } \\
\hline & $62.5 \%$ & $62.5 \%$ & \\
\hline \multirow{2}{*}{ ADHD (hyperactive/impulsive subtype) } & $N=6$ & $N=5$ & \multirow[b]{2}{*}{-} \\
\hline & $37.5 \%$ & $37.5 \%$ & \\
\hline \multirow{2}{*}{ ODD } & $N=8$ & $N=8$ & \multirow{2}{*}{-} \\
\hline & $50.0 \%$ & $50.0 \%$ & \\
\hline \multirow{2}{*}{ RAD } & $N=7$ & $N=7$ & \multirow{2}{*}{-} \\
\hline & $43.8 \%$ & $43.8 \%$ & \\
\hline \multirow{2}{*}{ RAD (inhibited subtype) } & $N=1$ & $N=1$ & \multirow[b]{2}{*}{ - } \\
\hline & $6.3 \%$ & $6.3 \%$ & \\
\hline \multirow{2}{*}{ RAD (disinhibited subtype) } & $N=6$ & $N=6$ & \multirow{2}{*}{-} \\
\hline & $37.5 \%$ & $37.5 \%$ & \\
\hline \multirow{2}{*}{ Specific phobia } & $N=7$ & $N=7$ & \multirow{2}{*}{ - } \\
\hline & $43.8 \%$ & $43.8 \%$ & \\
\hline \multirow{2}{*}{ MDD } & $N=5$ & $N=5$ & \multirow{2}{*}{ - } \\
\hline & $31.3 \%$ & $31.3 \%$ & \\
\hline \multirow{2}{*}{ SAD } & $N=5$ & $N=5$ & \multirow{2}{*}{ - } \\
\hline & $31.3 \%$ & $31.3 \%$ & \\
\hline \multirow{2}{*}{ Conduct disorder } & $N=3$ & $N=3$ & \multirow{2}{*}{ - } \\
\hline & $18.8 \%$ & $18.8 \%$ & \\
\hline \multirow{2}{*}{ Social phobia } & $N=1$ & $N=1$ & \multirow{2}{*}{-} \\
\hline & $6.3 \%$ & $6.3 \%$ & \\
\hline
\end{tabular}

Note. PTSD = Post Traumatic Stress Disorder, ADHD = Attention Deficit Hyperactivity Disorder, ODD = Oppositional Defiant Disorder, RAD = Reactive Attachment Disorder, $\mathrm{MDD}=$ Major Depressive Disorder, $\mathrm{GAD}=$ Generalized Anxiety Disorder, $\mathrm{OCD}=$ Obsessive Compulsive Disorder. Note. PTSD-AA = Post Traumatic Stress Disorder Alternative Algorithm for Young Children [20].

Problems with peers were found to have a mean score of 2.29 (SD 1.98) for boys and 4.11 (SD 1.76) for girls that states 12 (75.0\%) of the caregivers rated peer problems within clinical or borderline range. Conduct problems and ratings within clinical or borderline range were the case in 9 children (56.6\%) with a mean of 2.29 (SD 1.98) for boys and 4.11 (SD 1.76) for girls (Table 3).

Table 4 sets out mean sum scores and standard deviations for respectively the Danish 5 - 7-year old SDQ norm parent ratings and these high-risk sample caregiver ratings. The percentiles were also compared. The mean scores in the Danish norm data were found to significantly lower in all domains compared to this high-risk study sample. All children in this study had clinical or borderline scores (within the highest $20 \%$ of the norm range) in two or more domains.

\section{Discussion}

This study examined the assessment screening of trauma symptoms, psychopathology and psychosocial function in a high-risk sample of 4 - 8-year-old children 
Table 3. SDQ cut offs for Danish 5 - 7-year-olds parent ratings compared to the high-risk 4 - 7-year-olds caregiver ratings.

\begin{tabular}{|c|c|c|c|c|c|c|}
\hline \multirow[b]{2}{*}{5 - 7-year-olds boys } & \multicolumn{3}{|c|}{$\begin{array}{l}\text { Recommended bandings for boys } \\
\qquad(N=28,920)\end{array}$} & \multicolumn{3}{|c|}{$\begin{array}{l}\text { Recommended bandings for girls } \\
\qquad(N=27,611)\end{array}$} \\
\hline & Normal & Borderline & Clinical & Normal & Borderline & Clinical \\
\hline Total difficulties & $0-9$ & $10-13$ & $14-40$ & $0-8$ & $9-11$ & $12-14$ \\
\hline Emotional problems & $0-3$ & 4 & $5-10$ & $0-3$ & 4 & $5-10$ \\
\hline $\begin{array}{l}\text { Hyperactivity/ } \\
\text { Inattention }\end{array}$ & $0-4$ & $5-6$ & $7-10$ & $0-3$ & $4-5$ & $6-10$ \\
\hline Conduct problems & $0-2$ & 3 & & $0-2$ & 3 & $4-10$ \\
\hline \multirow[t]{2}{*}{ Peer problems } & $0-1$ & 2 & $4-10$ & $0-1$ & 2 & $3-10$ \\
\hline & \multicolumn{3}{|c|}{ Sample scores boy $(N=7)$} & \multicolumn{3}{|c|}{ Sample scores girls $(N=9)$} \\
\hline Caregiver ratings & Normal & Borderline & Clinical & Normal & Borderline & Clinical \\
\hline Total difficulties & $0-9$ & $10-13$ & $14-40$ & $0-8$ & $9-11$ & $12-14$ \\
\hline$N$ & - & - & $7 / 7$ & - & $1 / 9$ & $8 / 9$ \\
\hline Emotional problems & $0-3$ & 4 & $5-10$ & $0-3$ & 4 & $5-10$ \\
\hline$N$ & - & $2 / 7$ & $5 / 7$ & $2 / 9$ & - & $7 / 9$ \\
\hline $\begin{array}{l}\text { Hyperactivity/ } \\
\text { Inattention }\end{array}$ & $0-4$ & $5-6$ & $7-10$ & $0-3$ & $4-5$ & $6-10$ \\
\hline$N$ & - & $1 / 7$ & $6 / 7$ & $3 / 9$ & $2 / 9$ & $4 / 9$ \\
\hline Conduct problems & $0-2$ & 3 & $4-10$ & $0-2$ & 3 & $4-10$ \\
\hline$N$ & $1 / 7$ & - & $6 / 7$ & $6 / 9$ & - & $3 / 9$ \\
\hline Peer problems & $0-1$ & 2 & $3-10$ & $0-1$ & 2 & $3-10$ \\
\hline$N$ & $3 / 7$ & $1 / 7$ & $3 / 7$ & $1 / 9$ & - & $8 / 9$ \\
\hline
\end{tabular}

with a child protection case who were referred to counseling by the social authorities. First, psychopathology was present in all the children in this sample and the distribution of disorders showed an almost $100 \%$ prevalence of comorbidity among the children with the most common disorders being PTSD, ADHD inattentive subtype, ODD and RAD. Thus, comorbidity was the rule rather than the exception with 15/16 children meeting the criteria for at least three psychiatric diagnosis. Moreover, the results of SDQ indicated that all 16 children had a score of total difficulties above normal range when compared to a Danish norm population. Most profound was emotional symptoms and symptoms of hyperactivity and inattention. However, a great amount of the children also showed high ratings of peer problems and all 16 children had subscale scores within the borderline or clinical range in two or more domains. Thus, the prevalence of SDQ-measured mental health problems was relatively high in this sample when compared with a Danish norm population of $5-7$ boys and girls. The higher prevalence might reflect differences in psychosocial risk load and environmental stress given the social context.

Furthermore, all the children had been exposed to multiple traumas and had experienced at least three potential traumatic events prior to the age of six with a 
Table 4. Mean sum scores and Standard deviations for 5- and 7-year-old parent ratings compared to this high-risk 4 - 8-year-olds study sample.

\begin{tabular}{|c|c|c|c|c|c|c|c|c|}
\hline \multirow{3}{*}{$\begin{array}{c}\text { Caregiver rating } \\
\text { SDQ scale }\end{array}$} & \multicolumn{8}{|c|}{ High-risk 4 - 8-year-olds study sample } \\
\hline & \multicolumn{4}{|c|}{ Boys $(N=7)$} & \multicolumn{4}{|c|}{$\operatorname{Girls}(N=9)$} \\
\hline & \multicolumn{2}{|c|}{ Mean } & \multicolumn{2}{|c|}{ SD } & \multicolumn{2}{|c|}{ Mean } & \multicolumn{2}{|c|}{$\mathrm{SD}$} \\
\hline Total difficulties & \multicolumn{2}{|c|}{21.43} & \multicolumn{2}{|c|}{3.21} & \multicolumn{2}{|c|}{17.22} & \multicolumn{2}{|c|}{5.43} \\
\hline Emotional problems & \multicolumn{2}{|c|}{5.89} & \multicolumn{2}{|c|}{1.35} & \multicolumn{2}{|c|}{6.22} & \multicolumn{2}{|c|}{2.39} \\
\hline $\begin{array}{l}\text { Hyperactivity/ } \\
\text { Inattention }\end{array}$ & \multicolumn{2}{|c|}{8.43} & \multicolumn{2}{|c|}{1.27} & \multicolumn{2}{|c|}{4.88} & \multicolumn{2}{|c|}{3.22} \\
\hline Conduct problems & \multicolumn{2}{|c|}{4.86} & \multicolumn{2}{|c|}{1.46} & \multicolumn{2}{|c|}{2.00} & \multicolumn{2}{|c|}{2.45} \\
\hline \multirow[t]{2}{*}{ Peer problems } & \multicolumn{2}{|c|}{2.29} & \multicolumn{2}{|c|}{1.98} & \multicolumn{2}{|c|}{4.11} & \multicolumn{2}{|c|}{1.76} \\
\hline & \multicolumn{4}{|c|}{5 -year-olds $(N=3288)$} & \multicolumn{4}{|c|}{7 -year-olds $(N=53476)$} \\
\hline Parents rating & \multicolumn{2}{|c|}{ Boys } & \multicolumn{2}{|c|}{ Girls } & \multicolumn{2}{|c|}{ Boys } & \multicolumn{2}{|c|}{ Girls } \\
\hline SDQ scale & Mean & SD & Mean & SD & Mean & SD & Mean & SD \\
\hline Total difficulties & 6.60 & 4.97 & 5.61 & 4.26 & 6.41 & 4.77 & 5.44 & 4.16 \\
\hline Emotional problems & 1.58 & 1.73 & 1.59 & 1.75 & 1.59 & 1.75 & 1.66 & 1.76 \\
\hline $\begin{array}{l}\text { Hyperactivity/ } \\
\text { Inattention }\end{array}$ & 2.74 & 2.27 & 2.11 & 1.94 & 2.73 & 2.31 & 2.02 & 1.98 \\
\hline Conduct problems & 1.32 & 1.43 & 1.12 & 1.27 & 1.28 & 1.35 & 1.15 & 1.21 \\
\hline Peer problems & 0.97 & 1.57 & 0.79 & 1.33 & 0.82 & 1.36 & 0.63 & 1.13 \\
\hline
\end{tabular}

From Niclasen et al., (2012).

mean age of 1.8 year when exposed to the first potential trauma experience. The most frequently reported traumas in our sample included witnessing interpersonal violence and exposure to other types of interpersonal trauma. It is noteworthy, that all 16 children had been exposed to other forms of trauma not specified in the list of DSM-IV, and most common was neglect of care and separation trauma. This finding contributes to the ongoing debate within the child trauma field whether the DSM concept of trauma is too narrow, both in conceptualizing the types of experiences that can lead to trauma responses and the nature of those trauma responses [30]. Furthermore, results indicate that even though PTSD is the most prevalent disorder, caregivers reported high levels of psychiatric comorbidity in their children. This is in line with previous research showing that children exposed to interpersonal victimization often meet criteria for several psychiatric disorders besides PTSD [2].

Thus, the results shed light on the lively discussion about whether the current psychiatric nosology and multiple comorbid diagnoses are sufficiently accurate to describe many victimized children, since it can potentially be leading to both under treatment, overtreatment and inadequate treatment. For example, it should be considered whether the high prevalence of attention deficit disorders is better described as a camouflaged trauma reaction. According to [12] following exposure to interpersonal trauma, disturbances of attention and consciousness may manifest as dissociation, depersonalization, memory disturbance, disrupted executive functioning and an inability to concentrate regardless of whether 
the task evokes trauma reminders.

Moreover, the oppositional behavior symptoms and high prevalence of ODD in this sample could be comprehended considering the vulnerable family environment with potential chaotic surroundings, inappropriate caregiver model behavior and lack of social support. Thus, engaging in defiant behavior or other externalizing behavior may be seen as a way of acting out as a response to trauma exposures or to an attempt to remaster a sense of relational control after exposure to an interpersonal trauma [2]. Further, if the caregivers are traumatized or have severe social and mental health problems themselves they may not be able to help their children regulate their emotions and facilitate appropriate behavior outcomes in the child post trauma [10].

When children experience interpersonal traumas, they not only develop typical PTSD responses such as over generalized fear or maladaptive cognitions and hyperarousal, but they also often struggle with the loss of their primary attachment figure [30]. Research suggests that children who experience child adversity perceive their interpersonal relationships as less supportive and overall have smaller social support networks [31] [32]. Social support has been argued to act as a protective factor in coping following trauma. One theory explaining this perceived low social support, is that children with a child protection case, are more likely to come from overall complex dysfunctional family environments and if a child perceives their relationships to be unsupportive this can result in social withdrawal and peer rejection. Subsequently, this may add to increasing negative schematic beliefs and psychopathology [32]. Considering this, it is not surprising that many children in this high-risk sample displayed symptoms of reactive attachment disorder and showed high rates of peer problems according to SDQ.

Moreover, there have been various initiatives to evaluate whether a complex PTSD subtype versus a separate disorder (e.g. developmental trauma disorder) should be included for individuals who develop complex trauma outcomes related to childhood experiences of trauma. The current and previous diagnostic classification systems do not include a formal diagnosis of complex PTSD, however the International Classification of Diseases, 11th version (ICD-11; World Health Organization), due out in 2018, will include a complex PTSD diagnosis where individuals must have experienced chronic (typically interpersonal) trauma; and in addition to core PTSD features of intrusion, avoidance, and sense of threat. Individuals with complex PTSD must also exhibit prominent features of affective dysregulation, negative self-concept, and interpersonal disturbance which are pervasive and occur across various contexts and relationships regardless of proximity to traumatic reminders [33]. Thus, it should be considered whether the symptom complexity displayed among the children in this study could reflect chronic and severe coexisting problems with emotion regulation, impulse control, attention and cognition, dissociation, interpersonal relationships, and attributions [12] [33]. This makes sense considering that all children in this study had been exposed to multiple potential traumatic experiences very 
early in life and much research suggests that this has a profound impact on the developing brain [12] [34]. However, currently there exist no single instrument that assesses complex trauma outcomes in young children [8]. This emphasizes the need for new or better assessment methods for children with a history of potential complex developmental trauma.

In conclusion, there seems to be an overall similarity between the results of DIPA measures and the SDQ scores among the children in this high-risk sample. However, although the DIPA and SDQ measures performed similarly in this study, and showed comparable screening accuracy, further analysis is required to establish the extent to which the positive screens for each measure identify the same versus different psychopathology. This is not just determined by the cut-points employed for each measure. This is because the DIPA and SDQ are designed to screen for different forms of psychopathology. However, the total picture is that the children in this sample displayed a wide and complex profile of symptoms, comorbidity and poor psychosocial functioning with general high ratings of SDQ subscale scores and the total difficulties scores were rated high above the clinical cut off limit when compared to a Danish norm population. Nevertheless, it is not possible to infer the causes of the observed comorbid psychiatric disorders and impairments on psychosocial functioning, since data are only included from one point of time and the results are compared across groups. $\mathrm{Fu}$ ture studies should therefore consider a longitudinal cohort design. In this way, it would be possible to examine the pre-, peri- and post morbid factors among young children in high-risk of exposure to multiple traumatization.

\subsection{Strengths and Limitations}

One strength of this study was the systematic screening approach. In the period of data collection, we invited every family with a 4 - 8-year-old child who initiated counseling to participate in this study. However, although participation rates were high, representativity and selection bias must be considered before drawing any general conclusions from the results.

Another strength is that we had access to the complete child protection case files for all children referred to counseling by the social authorities. However, retrospective assessment by reading and screening the files for potential unreported traumas has clear methodological limitations. We had to rely on the information that was written in the records, without being able to confirm if the information was correct. We did not know if the children and their parents were asked about more details than were documented or if some details may have been left out.

We wished to study children at familial risk with an age sensitive clinical measure and a comprehensive and validated psychosocial functioning questionnaire to explore the characteristics of children at high-risk as a group. This is relevant when planning health services specially tailored for this group of vulnerable and often overlooked children and their families. These families are often 
struggling in several ways with the challenges of normal family life and with severe mental problems and abnormal discourse at the same time. This assessment screening study contributes and elucidates a limited research field in an ecological setting that could enable us to establish interventions and develop better strategies to detect and relieve these difficulties for children in a similar situation in the future and maybe also to prevent further development into severe mental illness.

However, results in this pilot study were based on a small sample and further investigation of this challenging, high-risk population of children is suggested. Nevertheless, this exploratory work provides further, albeit tentative, evidence for the need of further research and development of new strategies that can provide adequate conceptualization of these children's difficulties.

\subsection{Clinical Significance}

The findings presented may play an important role in supporting children who are at risk for complex trauma responses in mental health services and public family treatment facilities. Governments and child welfare services have a responsibility to identify those children in high-risk and the need for therapeutic services. This can be achieved through systematic screening procedure and monitoring of psychosocial difficulties among all children with a child protection case.

A systematic approach would be helpful for identifying children who need more extensive help and to determine the intervention. Such measures should be reliable and valid instruments that are not too time consuming for the staff, and they should reflect different perspectives, such as the clinician's evaluation and the parent report. Examples of such instruments could be SDQ, which measures psychosocial functioning as well as emotional and behavioral problems and the DIPA, which measures trauma symptoms and other age specific psychopathology. However, the results in this study questions whether these measures are enough when it comes to an adequate assessment of the profound difficulties and complex symptom profile experienced by this vulnerable group of young children. This emphasizes the need for future investigation and development of new tentative validated measures that assess complex trauma outcomes in children at high-risk.

\section{Acknowledgements}

We would like to thank the families and staff at the Center for Interventions of Children and Adolescents (CIBU) in Odense, Denmark, for all the help in promoting and carry through data collection and other important research activity regarding this study.

\section{Funding}

This study was supported by the Council of The Danish Victim Fund [\#17-91000060]. 


\section{Conflicts of Interest}

On behalf of all authors, the corresponding author states that there is no conflict of interest.

\section{References}

[1] Verlinden, E., Opmeer, B.C., Van Meijel, E.P.M., Beer, R., De Roos, C., Bicanic, I.A.E., et al. (2015) Enhanced Screening for Posttraumatic Stress Disorder and Comorbid Diagnoses in Children and Adolescents. European Journal of Psychotraumatology, 6, 26661. https://doi.org/10.3402/ejpt.v6.26661

[2] Løkkegaard, S.S., Egebæk, S.A.B. and Elklit, A. (2017) Are Trauma and Post-Traumatic Stress Disorder Connected to Psychiatric Comorbidity in Danish Pre-Schoolers? Journal of Child \& Adolescent Trauma, 10, 353-361.

http://link.springer.com/10.1007/s40653-017-0146-z https://doi.org/10.1007/s40653-017-0146-Z

[3] Teicher, M.H. and Samson, J.A. (2013) Childhood Maltreatment and Psychopathology: A Case for Ecophenotypic Variants as Clinically and Neurobiologically Distinct Subtypes. American Journal of Psychiatry, 170, 1114-1133.

https://doi.org/10.1176/appi.ajp.2013.12070957

[4] Werner, E.E. (1993) Risk, Resilience, and Recovery: Perspectives from the Kauai Longitudinal Study. Development and Psychopathology, 5, 503-515. https://doi.org/10.1017/S095457940000612X

[5] Cirulli, F. and Suchecki, D. (2010) Developmental Determinants of Sensitivity and Resistance to Stress: A Tribute to Seymour "Gig” Levine. Neuroscience \& Biobehavioral Reviews, 34, 781. https://doi.org/10.1016/j.neubiorev.2010.02.010

[6] Perry, P.R.A., Blakley, T.L., Baker, W.L. and Vigilante, D. (1995) Childhood Trauma, the Neurobiology of Adaptation, and "Use-Dependent" Development of the Brain: How "States" Become "Traits." Infant Mental Health Journal, 16, 271-291. https://doi.org/10.1002/1097-0355(199524)16:4<271::AID-IMHJ2280160404>3.0.C $\underline{\mathrm{O} ; 2-\mathrm{B}}$

[7] Purvis, K.B., Razuri, E.B., Howard, A.R.H., Call, C.D., DeLuna, J.H., Hall, J.S., et al. (2015) Decrease in Behavioral Problems and Trauma Symptoms among at-Risk Adopted Children Following Trauma-Informed Parent Training Intervention. Journal of Child \& Adolescent Trauma, 8, 201-210.

https://doi.org/10.1007/s40653-015-0055-y

[8] Denton, R., Frogley, C., Jackson, S., John, M. and Querstret, D. (2017) The Assessment of Developmental Trauma in Children and Adolescence: A Systematic Review. Clinical Child Psychology and Psychiatry, 22, 260-287. https://doi.org/10.1177/1359104516631607

[9] Christoffersen, M.N. (2013) The Prevalence of Four Types of Childhood Maltreatment in Denmark. Clinical Practice \& Epidemiology in Mental Health, 9, 149-156. https://doi.org/10.2174/1745017901309010149

[10] De Young, A.C., Kenardy, J.A. and Cobham, V.E. (2011) Trauma in Early Childhood: A Neglected Population. Clinical Child and Family Psychology Review, 14, 231-250. https://doi.org/10.1007/s10567-011-0094-3

[11] Cloitre, S.B.C., Herman, J.L., van der Kolk, B., Pynoos, R., Wang, J., et al. (2009) A Developmental Approach to Complex PTSD: Childhood and Adult Cumulative Trauma as Predictors of Symptom Complexity. Journal of Traumatic Stress, 22, 399-408. https://doi.org/10.1002/jts.20444 
[12] D’Andrea, W., Ford, J., Stolbach, B., Spinazzola, J. and van der Kolk, B.A. (2012) Understanding Interpersonal Trauma in Children: Why We Need a Developmentally Appropriate Trauma Diagnosis. American Journal of Orthopsychiatry, 82, 187-200. https://doi.org/10.1111/j.1939-0025.2012.01154.x

[13] Dubowitz, H., Thompson, R., Proctor, L., Metzger, R., Black, M.M., English, D., et al. (2016) Adversity, Maltreatment, and Resilience in Young Children. Academic Pediatrics, 16, 233-239. https://doi.org/10.1016/j.acap.2015.12.005

[14] Romine, C.B. and Reynolds, C.R. (2005) A Model of the Development of Frontal Lobe Functioning: Findings from a Meta-Analysis. Applied Neuropsychology, 12, 190-201. https://doi.org/10.1207/s15324826an1204_2

[15] Thorup, A.A.E., Jepsen, J.R., Ellersgaard, D.V., Burton, B.K., Christiani, C.J., Hemager, N., et al. (2015) The Danish High Risk and Resilience Study-VIA 7-A Cohort Study of 520 7-Year-Old Children Born of Parents Diagnosed with Either Schizophrenia, Bipolar Disorder or Neither of These Two Mental Disorders. BMC Psychiatry, 15, 233.

http://bmcpsychiatry.biomedcentral.com/articles/10.1186/s12888-015-0616-5 https://doi.org/10.1186/s12888-015-0616-5

[16] Schandorph Løkkegaard, S., Elmose, M. and Elklit, A. (2019) Validation of the Diagnostic Infant and Preschool Assessment in a Danish, Trauma-Exposed Sample of Young Children. Scandinavian Journal of Child and Adolescent Psychiatry and Psychology, 7, 39-51.

[17] Greger, H.K., Jozefiak, T. and Myhre, A.K. (2013) Child Abuse Management Units: How can Mental Health Services be Further Improved? Scandinavian Journal of Child and Adolescent Psychiatry and Psychology, 1, 51-62. https://doi.org/10.21307/sjcapp-2013-008

[18] Goodman, R. (1997) The Strengths and Difficulties Questionnaire: A Research Note. Journal of Child Psychology and Psychiatry, 38, 581-586. https://doi.org/10.1111/j.1469-7610.1997.tb01545.x

[19] Gigengack, M.R., van Meijel, E.P.M., Alisic, E. and Lindauer, R.J.L. (2015) Comparing Three Diagnostic Algorithms of Posttraumatic Stress in Young Children Exposed to Accidental Trauma: An Exploratory Study. Child and Adolescent Psychiatry and Mental Health, 9, 14. http://www.capmh.com/content/9/1/14 https://doi.org/10.1186/s13034-015-0046-7

[20] Scheeringa, M.S., Zeanah, C.H., Myers, L. and Putnam, F.W. (2003) New Findings on Alternative Criteria for PTSD in Preschool Children. The Journal of the American Academy of Child and Adolescent Psychiatry, 42, 561-570. https://doi.org/10.1097/01.CHI.0000046822.95464.14

[21] Copeland, W.E., Keeler, G., Angold, A. and Costello, E.J. (2007) Traumatic Events and Posttraumatic Stress in Childhood. Archives of General Psychiatry, 64, 577-584. https://doi.org/10.1001/archpsyc.64.5.577

[22] Scheeringa, M.S., Wright, M.J., Hunt, J.P. and Zeanah, C.H. (2006) Factors Affecting the Diagnosis and Prediction of PTSD Symptomatology in Children and Adolescents. American Journal of Psychiatry, 163, 644-651.

https://doi.org/10.1176/ajp.2006.163.4.644

[23] American Psychiatric Association, American Psychiatric Association (2013) Diagnostic and Statistical Manual of Mental Disorders: DSM-5. 5th Edition, American Psychiatric Association, Washington DC, 947 p. https://doi.org/10.1176/appi.books.9780890425596

[24] Luby, J.L., Heffelfinger, A.K., Mrakotsky, C., Hessler, M.J., Brown, K.M. and Hilde- 
brand, T. (2002) Preschool Major Depressive Disorder: Preliminary Validation for Developmentally Modified DSM-IV Criteria. Journal of the American Academy of Child \& Adolescent Psychiatry, 41, 928-937. https://doi.org/10.1097/00004583-200208000-00011

[25] Niclasen, J., Teasdale, T.W., Andersen, A-M.N., Skovgaard, A.M., Elberling, H. and Obel, C. (2012) Psychometric Properties of the Danish Strength and Difficulties Questionnaire: The SDQ Assessed for More than 70,000 Raters in Four Different Cohorts. PLoS ONE, 7, e32025. https://doi.org/10.1371/journal.pone.0032025

[26] Obel, C., Dalsgaard, S., Stax, H.-P. and Bilenberg, N. (2003) Strengths and Difficulties Questionnaire (SDQ-Dan). A New Instrument for Psychopathologic Screening of Children Aged 4-16 Years. Ugeskrift for Læger, 165, 462-465.

[27] Goodman, R. (2001) Psychometric Properties of the Strengths and Difficulties Questionnaire. The Journal of the American Academy of Child and Adolescent Psychiatry, 40, 1337-1345. https://doi.org/10.1097/00004583-200111000-00015

[28] Goemans, A., Tarren-Sweeney, M., van Geel, M. and Vedder, P. (2017) Psychosocial Screening and Monitoring for Children in Foster Care: Psychometric Properties of the Brief Assessment Checklist in a Dutch Population Study. Clinical Child Psychology and Psychiatry, 23, 9-24. https://doi.org/10.1177/1359104517706527

[29] Goodman, R. and Scott, S. (1999) Comparing the Strengths and Difficulties Questionnaire and the Child Behavior Checklist: Is Small Beautiful? Journal of Abnormal Child Psychology, 27, 17-24.

[30] Cohen, J.A., Mannarino, A.P. and Deblinger, E. (2017) Treating Trauma and Traumatic Grief in Children and Adolescents. 2nd Edition, The Guilford Press, New York, $356 \mathrm{p}$.

[31] Harmer, A.L., Sanderson, J. and Mertin, P. (1999) Influence of Negative Childhood Experiences on Psychological Functioning, Social Support, and Parenting for Mothers Recovering from Addiction. Child Abuse \& Neglect, 23, 421-433. https://doi.org/10.1016/S0145-2134(99)00020-4

[32] Murphy, S., Shevlin, M., Armour, C., Elklit, A. and Christoffersen, M.N. (2014) Childhood Adversity and PTSD Experiences: Testing a Multiple Mediator Model. Traumatology, 20, 225-231. https://doi.org/10.1037/h0099838

[33] Cloitre, G.D.W., Brewin, C.R., Bryant, R.A. and Maercker, A. (2013) Evidence for Proposed ICD-11 PTSD and Complex PTSD: A Latent Profile Analysis. European Journal of Psychotraumatology, 4, Article No. 20706. https://doi.org/10.3402/ejpt.v4i0.20706

[34] Kaffman, A. (2009) The Silent Epidemic of Neurodevelopmental Injuries. Biological Psychiatry, 66, 624-626. https://doi.org/10.1016/j.biopsych.2009.08.002 